Article

\title{
Contact-Free Optical Assessment of Changes in the Chest Wall Perfusion after Coronary Artery Bypass Grafting by Imaging Photoplethysmography
}

\author{
Imre Kukel ${ }^{1, \dagger}$, Alexander Trumpp $^{2}$, Katrin Plötze ${ }^{3}$, Antje Rost ${ }^{4}$, Sebastian Zaunseder ${ }^{5}{ }^{(D)}$, \\ Klaus Matschke ${ }^{3}$ and Stefan Rasche ${ }^{3,6, *, t}$ \\ 1 Interdisciplinary Intensive Care Unit, Neurology Clinic, Klinikum Bayreuth, 95445 Bayreuth, Germany; \\ imre.kukel@klinikum-bayreuth.de \\ 2 Institute of Biomedical Engineering, Faculty of Electrical and Computer Engineering, Technical University \\ Dresden, 01307 Dresden, Germany; alexandertrumpp@icloud.com \\ 3 Department of Cardiac Surgery, Heart Center Dresden, 01307 Dresden, Germany; \\ katrin.ploetze@herzzentrum-dresden.com (K.P.); klaus.matschke@herzzentrum-dresden.com (K.M.) \\ 4 Department of Anaesthesiology and Intensive Care Medicine, University Hospital Carl Gustav Carus, \\ Technical University Dresden, 01307 Dresden, Germany; antje.rost@uniklinikum-dresden.de \\ 5 Faculty of Information Technology, Dortmund University of Applied Sciences and Arts, \\ 44139 Dortmund, Germany; sebastian.zaunseder@fh-dortmund.de \\ 6 Center of Surgical Intensive Care Medicine, Department of Visceral, Thoracic and Vascular Surgery, \\ University Hospital Carl Gustav Carus, Technical University Dresden, 01307 Dresden, Germany \\ * Correspondence: stefan.rasche@ukdd.de; Tel.: +49-351-458-11521 \\ + I.K. and S.R. contributed equally to this manuscript.
}

Received: 24 August 2020; Accepted: 15 September 2020; Published: 18 September 2020

\begin{abstract}
Imaging photoplethysmography (iPPG) is a contact-free monitoring of the cutaneous blood volume pulse by RGB (red-green-blue) cameras. It detects vital parameters from skin areas and is associated to cutaneous perfusion. This study investigated the use of iPPG to quantify changes in cutaneous perfusion after major surgery. Patients undergoing coronary artery bypass grafting (CABG) were scanned before surgery and in three follow-up measurements. Using an industrial-grade RGB camera and usual indoor lighting, a contact-free imaging plethysmogram from the chest was obtained. Changes of the iPPG signal strength were evaluated in view of both the operation itself as well as the unilateral preparation of the internal thoracic artery (ITA) for coronary artery grafting, which is the main blood source of the chest wall. iPPG signal strength globally decreased after surgery and recovered partially during the follow up measurements. The ITA preparation led to a deeper decrease and an attenuated recovery of the iPPG signal strength compared to the other side of the chest wall. These results comply with the expected changes of cutaneous perfusion after CABG using an ITA graft. iPPG can be used to assess cutaneous perfusion and its global changes after major surgery as well as its local changes after specific surgical procedures.
\end{abstract}

Keywords: imaging photoplethysmography; cutaneous blood volume; cutaneous perfusion; coronary bypass operation; internal thoracic artery

\section{Introduction}

Imaging photoplethysmography (iPPG) is a contact-free monitoring approach based on the optical effects of the cutaneous circulation. The absorption and reflection of light at the skin is determined, among other factors, by its haemoglobin content and is thus subject to the pulsatile change of blood volume in the cutaneous and subcutaneous tissue [1]. This blood volume pulse causes tiny oscillations of the reflected light, which can be captured by conventional RGB cameras. 
iPPG has been shown to measure physiological data such as heart rate, heart rhythm and respiratory rate in experimental and clinical environments with a high accuracy [2-5]. As a special feature, its signal intensity responds directly to changes of the arterial blood pressure [6,7]. This haemodynamic sensitivity is linked to the peripheral vasculature of the skin, as the propagation of light into the tissue is limited to the outer skin layers. The iPPG signal is affected by autonomic nervous activity and vasomotor control in the peripheral vascular bed, such as in reactions to hot and cold thermal stimuli $[8,9]$, topically induced cutaneous vasodilation $[10,11]$, the vasodilatory effects of regional anaesthesia and reactive hyperaemia after vascular occlusion [11]. The blood volume pulse detected by iPPG is seen as an indirect measurement of skin perfusion $[1,12,13]$. This idea has been implemented in studies assessing burn wounds [14] and free flaps in plastic surgery, [15] or to reveal changes in the microcirculation of the skin associated with migraine $[10,16]$.

Skin perfusion is a valuable prognostic parameter and is used to guide the therapy of critically ill patients [17,18]. Clinical signs of a poor skin perfusion, like mottling or a prolonged capillary refill, indicate whole-body perfusion deficits during critical illness $[19,20]$, predict complications after major surgery [21] and are noted in treatment protocols for circulatory and septic shock [17,22]. Even when perceptible signs of cutaneous malperfusion are absent, a reduction of skin perfusion is common after major surgery as a result of neurohumoral responses, an increased activity of the sympathetic nervous system and a consecutive peripheral vasoconstriction.

In this study we investigated the suitability of iPPG to detect these typical changes in the microcirculation of the skin following major surgery. Patients undergoing coronary artery bypass grafting (CABG) with the use of a unilateral internal thoracic artery (ITA) graft were investigated. As the ITA is the main supplying vessel of the chest wall, the chest wall perfusion is inevitably affected after this procedure [23-25]. Hence, two issues are addressed: the global effect of major surgery on the peripheral circulation as well as the regional impact of ITA preparation on the chest wall perfusion.

\section{Materials and Methods}

\subsection{Study Setting and Patients}

A prospective, non-randomized observational study was carried out between December 2016 and April 2017 at the Department for Cardiac Surgery, Heart Center Dresden University Hospital. The study was approved by the Institutional Review Board of the Technical University Dresden (IRB00001473, EK168052013). The study was conducted in accordance with the principles of the fifth revision of the Declaration of Helsinki. Patients scheduled for elective coronary artery bypass surgery with the use of a unilateral ITA graft were included after informed consent was obtained. Patients undergoing emergency surgery or concomitant heart valve procedures were not investigated.

iPPGs were obtained from videos of the chest wall, which were recorded the day before surgery (baseline) and two hours (H2), one day (H24) and six (H144) days after surgery. For all patients, recordings at baseline and day six were done at the same place in an examination room. Recordings $\mathrm{H} 2$ and $\mathrm{H} 24$ were taken in the intensive care unit or the surgical ward at the bedside.

\subsection{Camera and Technical Setup}

An industrial grade CMOS camera (UI-3370CP-C- HQ, Imaging Development System GmbH, Obersulm Obersulm Germany) with a Cinegon 16/1.8 lens (Schneider Kreuznach, Bad Kreuznach, Bad Kreuznach Germany) was used. The camera was mounted to a custom-made mobile setup, allowing for a flexible and seamless integration of the measurements in the clinical workflow (Figure 1).

Videos were recorded with a spatial resolution of $420 \times 320$ pixels, a 12-bit colour depth, and a frame rate of 100 frames per second. Duration of the measurements was initially set to $60 \mathrm{~s}$. To achieve a higher data consistency, the measurements were extended to three minutes after four patients were examined. Videos were stored as a raw video on a computer and analysed offline (see below). 


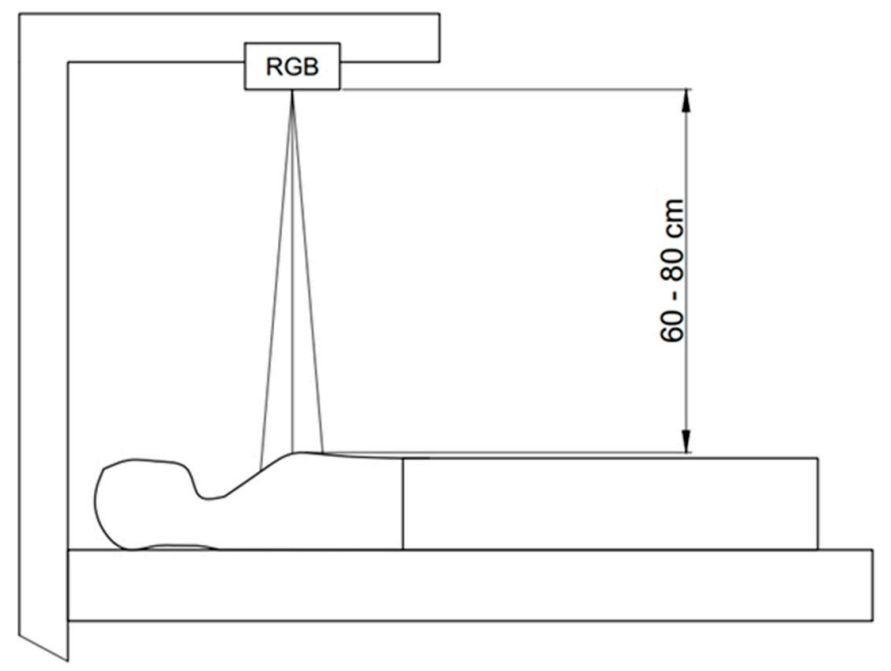

Figure 1. Schematic representation of the camera set-up.

A four-lead electrocardiogram (ECG) and a finger pulse oximetry were taken as reference from the bedside monitor (GE Healthcare Carescape Monitor B850) at sampling rates of $300 \mathrm{~Hz}$. The signals were synchronised with the video recordings.

No specific light source was used during the measurements. Experiments were conducted under usual ward lighting with fluorescent tubes and ambient light. The luminous colour, intensity and homogeneity of the illumination varied over the measurements, especially for $\mathrm{H} 2$ and $\mathrm{H} 24$. The robustness of the models used in the signal processing allows for minor variations caused by these effects, and their influence has been shown negligible in earlier studies [26,27].

\subsection{Recording Area and Region of Interest}

Patients assumed supine position during the recordings. The camera was positioned perpendicularly above the chest at a distance of 60 to $80 \mathrm{~cm}$. The camera was adjusted to cover the entire chest wall from the clavicular line to the lower line of the ribs (Figure 2). Regions of interest (ROI) were manually marked during the offline post-processing in each individual video by a dedicated selector in the analysis software. Areas of the wound dressing and ECG electrodes were excluded. To consider regional effects of ITA preparation on the skin perfusion, two corresponding ROIs were selected, one from the side of ITA preparation (ITA side) and one from the other side of the chest (Figure 2).

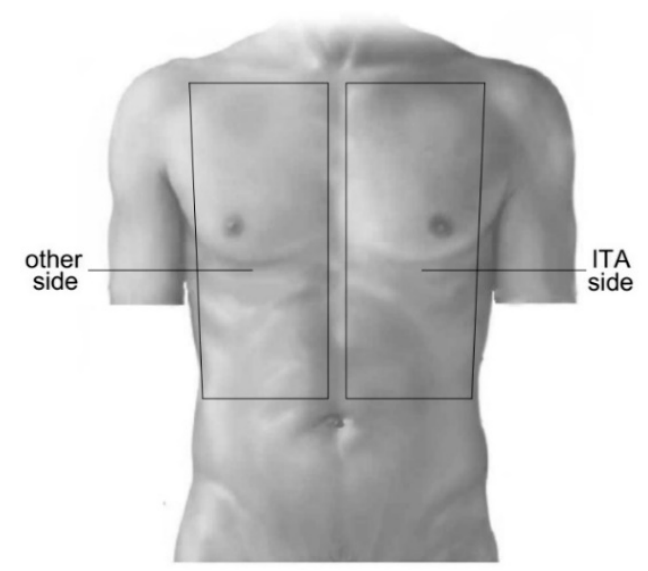

Figure 2. Recording area and regions of interest. 


\subsection{Signal Processing}

Data processing was done within a custom software framework, which allowed semiautomatic offline processing guided by the clinical investigator. The software was implemented in Matlab. The processing pipeline included multiple steps, which are briefly described below.

ROI definition: A custom graphical user interface allowed manual definition of ROIs. Details of the placement are as stated before. The ROIs were defined in the first frame and were kept in size and shape throughout the respective recording.

Image processing: To account for small movements of the thoracic region, we used optical flow to adapt the position of predefined ROIs.

Signal extraction: To derive iPPG signal courses, we averaged the pixels over each ROI using the green channel.

Signal processing: To account for a slightly inhomogeneous frame rate and eventually missing frames, we first interpolated iPPG signal courses to a sampling frequency of $100 \mathrm{~Hz}$ by Matlab's Piecewise Cubic Hermite Interpolating Polynomial. Afterwards, we detrended the signal courses and applied a 250 tap finite-impulse-response high-pass filter with a cutoff frequency of $0.5 \mathrm{~Hz}$ in forward and backward direction.

Feature extraction: We used the signal-to-noise ratio (SNR) as described in [28] to quantify the perfusion. The SNR is calculated in the frequency domain. It relates the power around the heart rate fHR and its first harmonic $2^{*} \mathrm{fHR}$ (we use fHR $\pm 5 \mathrm{bpm}$ and $2^{*} \mathrm{fHR} \pm 5 \mathrm{bpm}$ ) to the overall power in the frequency range between 30 and 200 beats per minute. The heart rate fHR was derived from the reference ECG. More details on the calculation of the SNR can be found in our previous works $[4,6]$. The SNR was calculated from signal excerpts of $10 \mathrm{~s}$. For each subject, a representative SNR of each side of the chest wall was derived by averaging all $10 \mathrm{~s}$ signal excerpts of a recording.

\subsection{Statistical Analysis}

The effects on iPPG of both the operation as well as the ITA preparation were evaluated in a mixed-effects model. Fixed effects were defined by the measurement time and the side of the chest wall. Measurement time was defined as an ordinal variable in relation to surgery. The side of the chest wall was used to assess the effects of ITA preparation. Random effects were defined by patient, and measurement time was nested at patient level.

Signal-to-noise ratio (SNR) values are given as estimated means and $95 \%$ confidence intervals (95 CI) derived from the model.

To account for any effects of age, sex, height, weight and heart rhythm on iPPG, a multivariable mixed-effects model was constructed and pared down on the basis of a stepwise deletion.

Models were compared by likelihood ratio (LR) tests. Significance of fixed effects was $t$-tested after Satterthwaite's approximations to degrees of freedom [29]. The significance level was set at $p<0.05$. Statistics were calculated with R, Version 3.6.1 [30].

\section{Results}

The study investigated 47 male and 2 female patients aged 69 (65-76) years. All patients were in sinus rhythm before surgery. Three patients were excluded after baseline measurements, since the surgical procedure was changed and no unilateral ITA graft was used.

The mean value of SNR overall measurements was $-1.80 \pm 0.08$ AU (arbitrary units, mean \pm standard error). The SNR was significantly affected by surgery (Figure 3, Table 1).

SNR values were highest at baseline and dropped persistently after surgery at both sides of the chest. The lowest SNR occurred at the measurement H2. A partial recovery of SNR was noted at H24 and H144. The SNR remained significantly below the baseline value until the last measurement H144 (Table 1). 


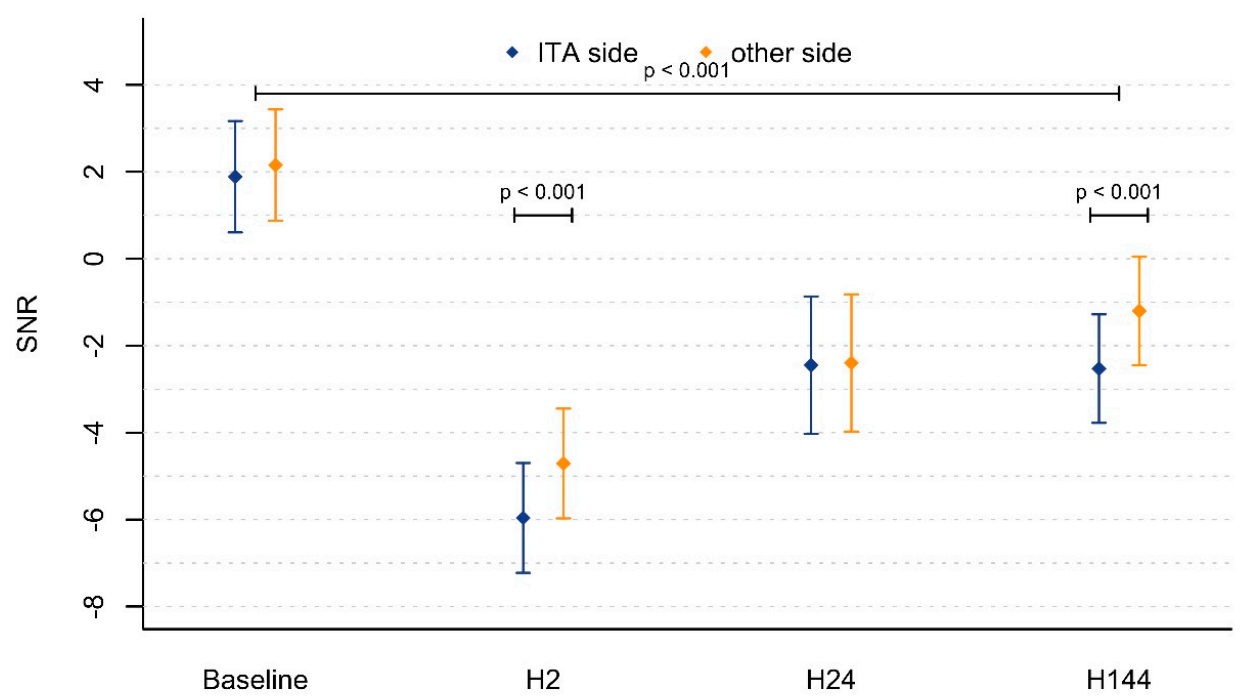

Figure 3. SNR values before (baseline) and two hours (H2), one day (H24) and six days (H144) after surgery. Means and 95\% confidence intervals are shown.

Table 1. Signal-to-noise ratio (SNR) in arbitrary units. 95\% CI: 95\% confidence interval. SNR was calculated before (BL) and two hours (H2), one day (H24) and six days (H144) after surgery. ITA side: side of the chest wall with preparation of the internal thoracic artery; other side: side of the chest wall without ITA preparation; $p_{1}$ for comparison against baseline; $p_{2}$ for the difference between both sides.

\begin{tabular}{ccccccc}
\hline & ITA Side & $\mathbf{9 5 \%} \mathbf{C I}$ & $\boldsymbol{p}_{\mathbf{1}}$ & Other Side & $\mathbf{9 5 \%}$ CI & $p_{\mathbf{2}}$ \\
\hline BL & 1.89 & $0.61-3.17$ & & 2.15 & $0.87-3.44$ & 0.121 \\
\hline H2 & -5.96 & $(-7.22)-(-4.70)$ & $<0.001$ & -4.71 & $(-5.97)-(-3.45)$ & $<0.001$ \\
\hline H24 & -2.45 & $(-4.03)-(-0.87)$ & $<0.001$ & -2.40 & $(-3.98)-(-0.82)$ & 0.427 \\
\hline H144 & -2.53 & $(-3.78)-(-1.28)$ & $<0.001$ & -1.20 & $(-2.45)-0.05$ & $<0.001$ \\
\hline
\end{tabular}

The use of ITA had a small but consistent effect on the perioperative response of SNR; $2.8 \%$ of the SNR variance was determined by ITA alone $\left(\sigma^{2}=8.33\right.$ vs. $\left.\sigma^{2}=8.57 \chi_{(\mathrm{df} 4)}^{2}=121, p<0.001\right)$.

Compared to baseline, SNR values decreased at $\mathrm{H} 2$ significantly more on the ITA side than on the other side of the chest wall ( $\Delta 7.85$ AU vs. $\Delta 6.86 \mathrm{AU}, p<0.001)$. Conversely, the partial recovery of SNR between $\mathrm{H} 2$ and $\mathrm{H} 24$ was higher on the ITA side ( $\Delta 3.52 \mathrm{AU}$ vs. $\Delta 2.31 \mathrm{AU}, p<0.001)$. At H24, SNR values of both sides were comparable (-2.45 AU vs. $-2.40 \mathrm{AU}, p=0.427)$. Only a nominal increase of SNR until H144 was observed on the chest wall side without ITA preparation $(\Delta 1.20$ AU vs. its preceding value at H24). Finally, SNR values remained significantly different at H144 (-2.53 AU vs. $-1.20 \mathrm{AU}$ for the ITA and the other side, respectively, $p<0.001$ ) (Tables 1 and 2).

Table 2. Different course of SNR at each side of the chest wall during the study. $p_{1}$ for ITA side; $p_{2}$ for the other side; $p_{3}$ for the difference in the course of between both sides.

\begin{tabular}{ccccccc}
\hline & ITA Side & $p_{\mathbf{1}}$ & Other Side & $p_{\mathbf{2}}$ & Side Difference & $p_{\mathbf{3}}$ \\
\hline BL to H2 & -7.85 & $<0.001$ & -6.86 & $<0.001$ & 0.985 & $<0.001$ \\
\hline H2 to H24 & 3.52 & $<0.001$ & 2.31 & $<0.05$ & -1.201 & $<0.001$ \\
\hline H24 to H144 & -0.08 & 0.937 & 1.20 & 0.306 & 1.277 & $<0.001$ \\
\hline
\end{tabular}

The effects of surgery and ITA preparation were independent from age, sex, weight and height of the patients. Heart rhythm returned to sinus rhythm in most patients at H24 and H144. Therefore, only the effects of atrial or ventricular pacing at $\mathrm{H} 2$ were tested, and no significant effect on SNR was found. 


\section{Discussion}

Contact-free photoplethysmograms were acquired by iPPG on the chest wall of patients before and after CABG. They showed an overall decrease of the signal strength after surgery, followed by a gradual but incomplete recovery until day six. They also indicated a specific impact of the unilateral ITA preparation on the perfusion of the respective side of the chest wall, which was illustrated by a deeper drop and slower recovery of the signal strength.

These results are consistent with the cardiovascular effects of major surgery on the cutaneous circulation, and the local outcome of ITA preparation on the perfusion of the chest wall. Depending on its extent, surgery initiates a reaction of the autonomous nervous system along with an upraise of circulating catecholamine levels and local vasoconstriction [31]. Aiming at the perfusion of vital organs, the cardiovascular reaction to a surgical trauma etiologically resembles that of shock to some degree [21]. In such conditions the peripheral circulation is the first to be limited and the last to be fully restored [18]. Accordingly, the number of perfused small vessels in the peripheral circulation is significantly reduced after cardiac surgery, even at a greater distance from the surgical site, and is not fully restored after $24 \mathrm{~h}$ [32]. The local effects of ITA preparation are demonstrated in a reduced sternal perfusion, which is partially balanced out by a collateral vascularization but is still evident seven days after surgery [33]. The ITA is also the main feeding vessel of the skin on the anterior chest wall with a restriction to the respective side of the body [25]. The resection of an ITA thus gives reason for a reduced cutaneous perfusion, though a collateral vascularization exists as well.

The relation of the iPPG signal to the microcirculatory sequelae after major surgery and to the local impact of ITA preparation is explained by its signal origin. The cutaneous blood volume pulse is seen as the key source of iPPG [34-36]. This is underlined by the peak signal strength of iPPG corresponding to the absorption maximum of haemoglobin in the spectral range of green light. Since the propagation of green light into the skin is circumscribed to about $1 \mathrm{~mm}[37,38]$, the hemodynamic information content of PPG is specific for the cutaneous microcirculation. Though not a direct measure, iPPG signals are strongly associated to cutaneous perfusion [13].

The results of this study prove iPPG is suitable to reveal the effects of major surgery on the perfusion of the skin. Remarkably, iPPG was able to reveal even small alterations of local blood supply accounting for only $2.8 \%$ of iPPG's signal variance. These outcomes depend on the specificity of iPPG for the cutaneous microcirculation, its sensitivity to autonomous nervous activity and vascular regulation and on the association of the cutaneous blood volume pulse to perfusion.

Of note, iPPG was evaluated by relative changes of its signal strength during the course of the study. Owing to their high variability between subjects, absolute values of iPPG parameters as presented here do not allow to draw conclusions about the actual amount of cutaneous perfusion. Further studies are needed to specify iPPG in its circulatory context and to clarify artefacts and confounders affecting the iPPG measurement.

\section{Conclusions}

iPPG can be used to assess the changes of cutaneous perfusion after major surgery. It is also sensitive for the effects of specific surgical procedures on regional cutaneous perfusion.

Author Contributions: Conceptualization, K.P., S.Z. and S.R.; Data curation, K.P., S.R.; Formal analysis, I.K., S.R.; Investigation, I.K. and A.R.; Project administration, K.M.; Resources, K.P. and S.Z.; Software, A.T. and S.Z.; Supervision, S.R.; Visualization, S.R.; Writing—original draft, I.K.; Writing-review \& editing, S.R. All authors have read and agreed to the published version of the manuscript.

Funding: This research received no external funding.

Acknowledgments: Open Access Funding by the Publication Fund of the TU Dresden.

Conflicts of Interest: The authors declare no conflict of interest. 


\section{References}

1. Huelsbusch, M.; Blazek, V. Contactless Mapping of Rhythmical Phenomena in Tissue Perfusion using PPGI. In Medical Imaging 2002: Physiology and Function from Multidimensional Images; International Society for Optics and Photonics: Bellingham, WA, USA, 2002; Volume 4683, pp. 110-117.

2. Aarts, L.A.; Jeanne, V.; Cleary, J.P.; Lieber, C.; Nelson, J.S.; Bambang Oetomo, S.; Verkruysse, W. Non-contact heart rate monitoring utilizing camera photoplethysmography in the neonatal intensive care unit-A pilot study. Early Hum. Dev. 2013, 89, 943-948. [CrossRef]

3. Couderc, J.P.; Kyal, S.; Mestha, L.K.; Xu, B.; Peterson, D.R.; Xia, X.; Hall, B. Detection of atrial fibrillation using contactless facial video monitoring. Heart Rhythm 2015, 12, 195-201. [CrossRef] [PubMed]

4. Rasche, S.; Trumpp, A.; Waldow, T.; Gaetjen, F.; Plotze, K.; Wedekind, D.; Schmidt, M.; Malberg, H.; Matschke, K.; Zaunseder, S. Camera-based photoplethysmography in critical care patients. Clin. Hemorheol. Microcirc. 2016, 64, 77-90. [CrossRef] [PubMed]

5. Tarassenko, L.; Villarroel, M.; Guazzi, A.; Jorge, J.; Clifton, D.A.; Pugh, C. Non-contact video-based vital sign monitoring using ambient light and auto-regressive models. Physiol. Meas. 2014, 35, 807-831. [CrossRef]

6. Rasche, S.; Trumpp, A.; Schmidt, M.; Plotze, K.; Gatjen, F.; Malberg, H.; Matschke, K.; Rudolf, M.; Baum, F.; Zaunseder, S. Remote Photoplethysmographic Assessment of the Peripheral Circulation in Critical Care Patients Recovering From Cardiac Surgery. Shock 2019, 52, 174-182. [CrossRef] [PubMed]

7. Trumpp, A.; Rasche, S.; Wedekind, D.; Rudolf, M.; Malberg, H.; Matschke, K.; Zaunseder, S. Relation between pulse pressure and the pulsation strength in camera-based photoplethysmograms. Curr. Dir. Biomed. Eng. 2017, 3, 489. [CrossRef]

8. Trumpp, A.; Schell, J.; Malberg, H.; Zaunseder, S. Vasomotor assessment by camera-based photoplethysmography. Curr. Dir. Biomed. Eng. 2016, 2, 199. [CrossRef]

9. Volynsky, M.A.; Margaryants, N.B.; Mamontov, O.V.; Kamshilin, A.A. Contactless Monitoring of Microcirculation Reaction on Local Temperature Changes. Appl. Sci. 2019, 9, 4947. [CrossRef]

10. Kamshilin, A.A.; Volynsky, M.A.; Khayrutdinova, O.; Nurkhametova, D.; Babayan, L.; Amelin, A.V.; Mamontov, O.V.; Giniatullin, R. Novel capsaicin-induced parameters of microcirculation in migraine patients revealed by imaging photoplethysmography. J. Headache Pain 2018, 19, 43. [CrossRef]

11. Marcinkevics, Z.; Rubins, U.; Zaharans, J.; Miščuks, A.; Urtane, E.; Ozolina-Moll, L. Imaging photoplethysmography for clinical assessment of cutaneous microcirculation at two different depths. J. Biomed. Opt. 2016, 21, 035005. [CrossRef]

12. Zheng, J.; Hu, S.; Echiadis, A.; Azorin-Peris, V.; Shi, P.; Chouliaras, V. A Remote Approach to Measure Blood Perfusion from the Human Face. In Advanced Biomedical and Clinical Diagnostic Systems VII; International Society for Optics and Photonics: Bellingham, WA, USA, 2009; Volume 7169, p. 716917.

13. Rasche, S.; Huhle, R.; Junghans, E.; Abreu, M.G.D.; Ling, Y.; Trumpp, A.; Zaunseder, S. Association of remote imaging photoplethysmography and cu-taneous perfusion in volunteers. Sci. Rep. in press.

14. Mo, W.; Mohan, R.; Li, W.; Zhang, X.; Sellke, E.; Fan, W.; DiMaio, J.M.; Thatcher, J. The Importance of Illumination in a Non-Contact Photoplethysmography Imaging System for Burn Wound Assessment. In Photonic Therapeutics and Diagnostics XI; International Society for Optics and Photonics: Bellingham, WA, USA, 2015; Volume 9303, p. 93030M.

15. Secerbegovic, A.; Mesic, H.; Bergsland, J.; Balasingham, I. Contactless Blood Perfusion Assessment of the Free Flap in Breast Reconstruction Surgery. In Proceedings of the 2019 13th International Symposium on Medical Information and Communication Technology (ISMICT), Oslo, Norway, 8-10 May 2019; pp. 1-4.

16. Zaproudina, N.; Teplov, V.; Nippolainen, E.; Lipponen, J.A.; Kamshilin, A.A.; Närhi, M.; Karjalainen, P.A.; Giniatullin, R. Asynchronicity of Facial Blood Perfusion in Migraine. PLoS ONE 2013, 8, e80189. [CrossRef] [PubMed]

17. Hernandez, G.; Ospina-Tascon, G.A.; Damiani, L.P.; Estenssoro, E.; Dubin, A.; Hurtado, J.; Friedman, G.; Castro, R.; Alegria, L.; Teboul, J.L.; et al. Effect of a Resuscitation Strategy Targeting Peripheral Perfusion Status vs. Serum Lactate Levels on 28-Day Mortality Among Patients With Septic Shock: The ANDROMEDA-SHOCK Randomized Clinical Trial. JAMA 2019, 321, 654-664. [CrossRef] [PubMed]

18. Lima, A.; Takala, J. Clinical significance of monitoring perfusion in non-vital organs. Intensive Care Med. 2014, 40, 1052-1054. [CrossRef] 
19. Ait-Oufella, H.; Bige, N.; Boelle, P.Y.; Pichereau, C.; Alves, M.; Bertinchamp, R.; Baudel, J.L.; Galbois, A.; Maury, E.; Guidet, B. Capillary refill time exploration during septic shock. Intensive Care Med. 2014, 40, 958-964. [CrossRef]

20. Lima, A.; Jansen, T.C.; Van Bommel, J.; Ince, C.; Bakker, J. The prognostic value of the subjective assessment of peripheral perfusion in critically ill patients. Crit. Care Med. 2009, 37, 934-938. [CrossRef]

21. Van Genderen, M.E.; Paauwe, J.; De Jonge, J.; Van der Valk, R.J.; Lima, A.; Bakker, J.; Van Bommel, J. Clinical assessment of peripheral perfusion to predict postoperative complications after major abdominal surgery early: A prospective observational study in adults. Crit. Care 2014, 18, R114. [CrossRef]

22. Dunser, M.W.; Takala, J.; Brunauer, A.; Bakker, J. Re-thinking resuscitation: Leaving blood pressure cosmetics behind and moving forward to permissive hypotension and a tissue perfusion-based approach. Crit. Care 2013, 17, 326. [CrossRef]

23. Boodhwani, M.; Lam, B.K.; Nathan, H.J.; Mesana, T.G.; Ruel, M.; Zeng, W.; Sellke, F.W.; Rubens, F.D. Skeletonized internal thoracic artery harvest reduces pain and dysesthesia and improves sternal perfusion after coronary artery bypass surgery: A randomized, double-blind, within-patient comparison. Circulation 2006, 114, 766-773. [CrossRef]

24. Kamiya, H.; Akhyari, P.; Martens, A.; Karck, M.; Haverich, A.; Lichtenberg, A. Sternal microcirculation after skeletonized versus pedicled harvesting of the internal thoracic artery: A randomized study. J. Thorac. Cardiovasc. Surg. 2008, 135, 32-37. [CrossRef]

25. Torregrossa, G.; Sosin, M.; Gerosa, G.; Tuffaha, S.H.; Gottlieb, L.J.; Christy, M.R.; Dorafshar, A.H. Chest Wall, Thymus, and Heart Transplant: Pushing the Boundary of Solid Organ and Vascularized Composite Allotransplantation. Vasc. Compos. Allotransplant. 2015, 2, 29-36. [CrossRef]

26. Sun, Y.; Azorin-Peris, V.; Kalawsky, R.; Hu, S.; Papin, C.; Greenwald, S. Use of ambient light in remote photoplethysmographic systems: Comparison between a high-performance camera and a low-cost webcam. J. Biomed. Opt. 2012, 17, 037005. [CrossRef] [PubMed]

27. Verkruysse, W.; Svaasand, L.O.; Nelson, J.S. Remote plethysmographic imaging using ambient light. Opt. Express 2008, 16, 21434-21445. [CrossRef]

28. Haan, G.D.; Jeanne, V. Robust Pulse Rate From Chrominance-Based rPPG. IEEE Trans. Biomed. Eng. 2013, 60, 2878-2886. [CrossRef] [PubMed]

29. Kuznetsova, A.; Brockhoff, P.B.; Christensen, R.H.B. lmerTest Package: Tests in Linear Mixed Effects Models. J. Stat. Softw. 2017, 82, 26. [CrossRef]

30. R Core Team. R: A Language and Environment for Statistical Computing; R Foundation for Statistical Computing: Vienna, Austria, 2019.

31. Desborough, J.P. The stress response to trauma and surgery. Br. J. Anaesth. 2000, 85, 109-117. [CrossRef] [PubMed]

32. De Backer, D.; Dubois, M.J.; Schmartz, D.; Koch, M.; Ducart, A.; Barvais, L.; Vincent, J.L. Microcirculatory alterations in cardiac surgery: Effects of cardiopulmonary bypass and anesthesia. Ann. Thorac. Surg. 2009, 88, 1396-1403. [CrossRef] [PubMed]

33. Carrier, M.; Grégoire, J.; Tronc, F.; Cartier, R.; Leclerc, Y.; Pelletier, L.C. Effect of internal mammary artery dissection on sternal vascularization. Ann. Thorac. Surg. 1992, 53, 115-119. [CrossRef]

34. Moço, A.V.; Stuijk, S.; De Haan, G. New insights into the origin of remote PPG signals in visible light and infrared. Sci. Rep. 2018, 8, 8501. [CrossRef]

35. Trumpp, A.; Bauer, P.L.; Rasche, S.; Malberg, H.; Zaunseder, S. The value of polarization in camera-based photoplethysmography. Biomed. Opt. Express 2017, 8, 2822-2834. [CrossRef]

36. Zaunseder, S.; Trumpp, A.; Wedekind, D.; Malberg, H. Cardiovascular assessment by imaging photoplethysmography-A review. Biomed. Tech. 2018, 63, 617-634. [CrossRef] [PubMed]

37. Bashkatov, A.N.; Genina, E.A.; Kochubey, V.I.; Tuchin, V.V. Optical properties of human skin, subcutaneous and mucous tissues in the wavelength range from 400 to $2000 \mathrm{~nm}$. J. Phys. D Appl. Phys. 2005, 38, 2543-2555. [CrossRef]

38. Braverman, I.M. The cutaneous microcirculation: Ultrastructure and microanatomical organization. Microcirculation 1997, 4, 329-340. [CrossRef] [PubMed]

(C) 2020 by the authors. Licensee MDPI, Basel, Switzerland. This article is an open access article distributed under the terms and conditions of the Creative Commons Attribution (CC BY) license (http://creativecommons.org/licenses/by/4.0/). 